\title{
Panorama dos gastos públicos municipais com os serviços de limpeza urbana e manejo de resíduos sólidos: uma análise da Região Metropolitana de Belo Horizonte (MG)
}

Overview of municipal public spending on urban cleaning services and solid waste management: an analysis of the Metropolitan Region of Belo Horizonte (MG)

\section{Ana Carla Ferreira** ${ }^{10}$, Raphael Tobias Vasconcelos Barros ${ }^{1}[\mathbb{C}$}

\section{RESUMO}

A crescente geração de resíduos sólidos urbanos é um problema que atinge países de diversos níveis de desenvolvimento. A falta de adequados planejamento e gerenciamento de resíduos sólidos urbanos acarreta prejuízos à qualidade de vida da população e ao ambiente e elevação dos custos do manejo desses resíduos. O presente trabalho apresenta o panorama dos gastos públicos municipais despendidos entre 2009 e 2017 com os serviços de limpeza urbana de 31 dos 34 municípios que compõem a Região Metropolitana de Belo Horizonte. Os municípios foram ordenados por faixas populacionais, conforme classifica o Instituto Brasileiro de Geografia e Estatística. Realizou-se análise estatística descritiva, e a normalidade dos dados foi testada (teste de Shapiro-Wilk; $\alpha=5 \%$ ). Também avaliou-se a existência de correlação entre as receitas e as despesas municipais com o manejo de resíduos sólidos urbanos (teste de Spearman; $\alpha=5 \%$ ). Verificouse, ainda, a existência de diferenças significativas entre grupos de municípios em função do agrupamento por faixas populacionais (teste de Kruskal-Wallis; $\alpha=5 \%$ ). Os resultados apontaram diferenças significativas do custo per capita despendido pelos municípios com os serviços de manejo de resíduos sólidos urbanos entre as seis faixas populacionais comparadas. Os gastos municipais relativos à faixa populacional "acima de 500.000 habitantes" foram significativamente superiores aos das demais faixas. Embora não discuta qualidade e cobertura dos serviços, este trabalho contribui para a área, visto a escassez de estudos envolvendo custos e resíduos sólidos urbanos, bem como possibilita a criação de estratégias direcionadas à otimização dos gastos públicos visando à sustentabilidade financeira.

Palavras-chave: manejo de resíduos sólidos urbanos; serviços municipais; custos; Região Metropolitana de Belo Horizonte.

\begin{abstract}
The growing generation of urban solid waste (USW) undistinctively affects countries having different levels of development. The lack of adequate planning and management of USW causes damage to the quality of life of the population and to the environment and increases the costs of handling this waste. This work is aimed to present a panorama of municipal public expenditures between 2009 and 2017 on urban cleaning services in 31 out of 34 municipalities comprising the Metropolitan Region of Belo Horizonte, Brazil. The municipalities were ordered by population groups, according to the classification of the Brazilian Institute of Geography and Statistics. A descriptive statistical analysis was performed and the normality of the data was tested (Shapiro-Wilk test; $\alpha=5 \%$ ). The existence of a correlation between municipal revenues and expenses relative to the management of USW was also evaluated (Spearman test; $\alpha=5 \%$ ). The existence of significant differences among groups of municipalities due to the grouping by population (Kruskal-Wallis test; $\alpha=5 \%$ ) was also verified. In general, the results showed significant differences in the per capita cost spent by municipalities with SUW management services among the six population groups compared. Municipal expenditures for the "over 500,000 inhabitants" population group were significantly higher than for other groups. Although it does not discuss quality and extension of the services, this work contributes to the area, given the scarcity of studies involving costs and SUW, as well as enables the creation of strategies aimed at optimizing public spending aimed at financial sustainability.
\end{abstract}

Keywords: management of solid urban waste; municipal services; costs; Metropolitan Region of Belo Horizonte.

'Departamento de Engenharia Sanitária e Ambiental, Escola de Engenharia, Universidade Federal de Minas Gerais - Belo Horizonte (MG), Brasil.

*Autora correspondente: anacarlanevesferreira@gmail.com

Conflitos de interesse: os autores declaram não haver conflito de interesses.

Financiamento: nenhum.

Recebido: 25/01/2020 - Aceito: 07/07/2020 - Reg. ABES: 20200022 


\section{INTRODUÇÃO}

A problemática que envolve a gestão de resíduos sólidos urbanos (RSU) tem se tornado cada vez mais crítica, principalmente, pelo aumento do consumo e da consequente produção de RSU. A postura da sociedade contemporânea em relação à utilização crescente de produtos com menores ciclos de vida e de embalagens descartáveis também tem contribuído para a situação de precariedade da gestão de RSU, na qual se destaca seu inadequado gerenciamento como um dos principais problemas ambientais e sanitários do mundo (BARROS, 2012; GONÇALVES; TANAKA; AMEDOMAR, 2013).

Embora o desenvolvimento urbano seja um dos condicionantes de uma gestão pública eficiente, a urbanização acelerada e sem planejamento tem como consequência problemas de ordem ambiental e social, destacando-se o aumento do volume de RSU gerados e a precariedade dos serviços públicos de manejo (VASCONCELOS et al., 2016). Segundo a Associação Brasileira de Empresas de Limpeza Pública e Resíduos Especiais (ABRELPE), foram geradas aproximadamente 71,6 milhões de toneladas de RSU no Brasil em 2017, o que representa $0,76 \mathrm{~kg} / \mathrm{hab}_{\text {.dia }}{ }^{-1}$. Além disso, 59,1\% dos RSU coletados são destinados aos lixões ou aos aterros controlados, destinos estes que não dispõem de medidas necessárias para a proteção ao meio ambiente (ABRELPE, 2017).

Diversos impactos à saúde e ao meio ambiente associam-se à indevida gestão de RSU, entre os quais se destacam a proliferação de vetores de doenças, o acúmulo de resíduos e consequente poluição visual das vias públicas, os alagamentos das vias em decorrência da obstrução dos sistemas de drenagem, a contaminação dos solos e dos cursos d'água e as doenças respiratórias, além de dengue e disfunções gastrointestinais (HOORNWEG; Bhada-Tata, 2012). Destaca-se que a falta de adequados planejamento e gerenciamento de resíduos sólidos acarreta não só em prejuízos à qualidade de vida da população e ao ambiente, mas em elevação de custos com o manejo desses resíduos (BARROS, 2012).

No Brasil, entrou em vigor a Lei no 12.305 , de 2 de agosto de 2010, que instituiu a Política Nacional de Resíduos Sólidos (PNRS) (BRASIL, 2010). Nessa lei, estabeleceram-se metas e prioridades associadas à melhoria da gestão dos RSU, as quais refletem um inevitável aumento dos custos e investimentos com o manejo de RSU. Segundo a Secretaria do Tesouro Nacional (STN), a gestão da limpeza urbana está entre o terceiro e o quarto maior gasto das gestões municipais nacionais (STN, 2019). Estima-se que os municípios brasileiros tenham despendido, em média, R\$124,44/ano per capita com os serviços de limpeza urbana (ABRELPE, 2017).

No estado de Minas Gerais, diversos municípios apresentam dificuldades em relação à manipulação de RSU e realizam, muitas vezes, o depósito desses resíduos em vazadouros a céu aberto (lixões) - destino potencialmente danoso e prejudicial ao meio ambiente (BARROS; SILVA; MIRANDA, 2005; BARROS, 2012; FEAM, 2016). Segundo o Plano Municipal de Saneamento de Belo Horizonte (MG), a produção per capita de RSU na capital do estado acompanhou o crescimento observado em nível nacional e aumentou em 27,2\% entre 2003 e 2015 (BELO HORIZONTE, 2016).

Segundo a Agência de Desenvolvimento da Região Metropolitana de Belo Horizonte (2018), a Região Metropolitana de Belo Horizonte (RMBH), composta de 34 municípios mineiros, é considerada a sétima maior região metropolitana da América Latina, e os gastos desses municípios com a gestão dos RSU representam uma parcela expressiva dos gastos de Minas Gerais com esses resíduos. Segundo o Plano Metropolitano de Resíduos Sólidos da RMBH e Colar Metropolitano (MINAS GERAIS, 2013), a RMBH e o Colar
Metropolitano (composto de outros 16 municípios mineiros) geraram, juntos, cerca de 4.600 t/dia de RSU em 2010. Em relação à gestão de RSU desses municípios, os custos com transportes são apontados como o principal constituinte nos custos totais de RSU (MINAS GERAIS, 2013).

Custos dos serviços de limpeza pública e de manejo de resíduos sólidos (RS) têm sido raramente abordados nos trabalhos sobre regiões metropolitanas. O Sistema Nacional de Informações Sobre Saneamento (SNIS, 2019) apresenta uma avaliação de acordo com as regiões brasileiras. Em 2019, os valores médios das despesas per capita com os serviços de manejo de RS apresentaram variação máxima de cerca de $30 \%$ entre as regiões, sendo o valor mínimo de R\$98,42/habitante/ano, na Região Norte, e o valor máximo de R\$144,62/habitante/ano, na Região Sudeste.

Sobre a cobrança pelos serviços de coleta, transporte e destinação final de RSU, o SNIS (2019) também aponta uma diferença entre os valores apresentados entre as regiões brasileiras: a Região Nordeste é a que possui o menor percentual de cobrança pelos municípios (9,1\%), seguida da Região Norte (20,6\%), da Região Centro-oeste (28,0\%), da Região Sudeste (50,8\%) e da Região Sul, com o maior percentual de cobrança $(85,4 \%)$.

Ressalta-se a existência de poucos estudos na literatura acerca dos custos que envolvem o manejo de RSU. Conceição et al. (2012), Conceição et al. (2016) e Simões e Marques (2012) concluíram que apenas 0,63\% das publicações dos principais congressos brasileiros abordam a temática resíduos e custos. Assim, a consideração do presente trabalho poderá contribuir para o fomento dos escassos estudos sobre custos envolvendo tanto os serviços de limpeza pública quanto o manejo de RSU. Em uma perspectiva social, o estudo também poderá colaborar com os gestores de políticas públicas quanto ao posicionamento da situação dos gastos com RSU e à identificação dos fatores que influenciam esses custos. Isso possibilitará a criação de estratégias direcionadas à redução dos gastos públicos para realizar os referidos serviços.

Finalmente, esta pesquisa se justifica, ainda, pela relevância e aplicação socioeconômica da PNRS e da Política Estadual de Resíduos Sólidos (PERS) que estabelecem como princípio a sustentabilidade financeira da prestação dos serviços de limpeza urbana e manejo de RSU (MINAS GERAIS, 2009; BRASIL, 2010). Nesse contexto, o presente trabalho visou analisar, por meio de ferramentas estatísticas, dados dos municípios que compõem a RMBH, no período entre 2009 e 2017, referentes aos gastos públicos municipais despendidos com serviços de limpeza urbana.

\section{METODOLOGIA}

\section{Área de estudo}

A RMBH, regulamentada pela Lei Estadual nº 6.303, de 1974, está localizada no estado de Minas Gerais e é formada por 34 municípios, possuindo uma população de 5.873 .841 habitantes, distribuídos em uma área de $9.460 \mathrm{~km}^{2}$ (RMBH, 2018).

Os 31 municípios que compõem a RMBH, cujos dados apresentaram-se disponíveis para o presente estudo (não foram obtidos dados para os municípios Confins, Itatiaiuçu e Taquaraçu de Minas), foram ordenados em seis diferentes faixas populacionais (portes) conforme a divisão estabelecida pelo Instituto Brasileiro de Geografia e Estatística (IBGE), sendo o maior percentual de municípios (29\%) atribuído à faixa populacional de 20.001 a 50.000 habitantes e o menor $(6,5 \%)$ correspondente à faixa acima de 500.000 habitantes (Figura 1) 
Ressalta-se que foram considerados os dados relativos a 2018 (os mais recentes) para a referida ordenação.

\section{Indicadores avaliados}

Utilizaram-se dados secundários de indicadores relativos às receitas e às despesas municipais, incluindo-se aqueles referentes ao manejo de RSU (Quadro 1), provenientes do SNIS e da STN para 31 municípios da RMBH, no período de 2009 a 2017.

Consideraram-se os valores deflacionados médios anuais (2009 a 2017), segundo o Índice Nacional de Preços ao Consumidor Amplo (IPCA), para realizar a correção financeira de todos os valores referentes às receitas e às despesas avaliadas, tendo como ano-base 2018. Para obter os dados per capita, utilizaram-se as estimativas populacionais de cada ano, conforme as informações provenientes do IBGE (2019).

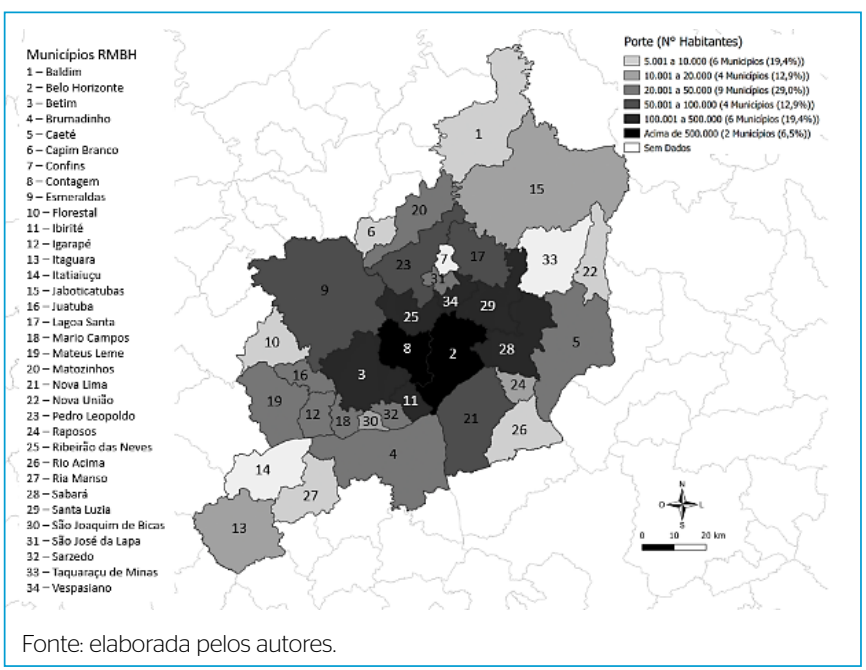

Figura 1 - Municípios da Região Metropolitana de Belo Horizonte $(n=34)$, ordenação por faixas populacionais $(n=6)$ conforme o Instituto Brasileiro de Geografia e Estatística e quantitativo e percentual de municípios pertencentes a cada respectivo porte.

Quadro 1 - Indicadores avaliados $(n=8)$ relativos às receitas $(n=2)$ e às despesas $(n=6)$ municipais, considerando o periodo entre 2009 e 2017.

\begin{tabular}{|l|c|}
\hline Receitas' (R\$) & Fonte \\
\hline Receita total per capita arrecadada pelo município & STN e IBGE \\
\hline $\begin{array}{l}\text { Receita per capita arrecadada com taxas e tarifas referentes à } \\
\text { gestão e manejo de RSU }\end{array}$ & SNIS \\
\hline Despesas' (R\$) & Fonte \\
\hline Despesa total com o manejo de RSU & SNIS \\
\hline Despesa per capita com o manejo de RSU & SNIS IBGE \\
\hline $\begin{array}{l}\text { Despesa total com o serviço de coleta de resíduos domiciliares } \\
\text { (RDO) e resíduos públicos (RPU) }\end{array}$ & SNIS \\
\hline Despesa total com os resíduos de serviço de saúde (RSS) & SNIS \\
\hline Despesa total com os serviços de varrição de logradouros públicos & SNIS \\
\hline $\begin{array}{l}\text { Despesa total com todos os agentes executores dos demais } \\
\text { serviços quando não especificados em campos próprios (outros) }\end{array}$ & SNIS \\
\hline
\end{tabular}

'Todos os valores monetários foram corrigidos conforme o Índice Nacional de Preços ao Consumidor Amplo (IPCA), considerando-se o ano-base de 2018; SNIS: Sistema Nacional de Informações Sobre Saneamento; STN: Secretaria de Tesouro Nacional; IBGE: Instituto Brasileiro de Geografia e Estatística; RSU: resíduos sólidos urbanos

\section{Análises estatísticas}

Inicialmente, os dados referentes aos indicadores de despesas e receitas municipais foram tabulados em planilhas no Excel (versão 2013). Em seguida, a normalidade dos dados foi testada por meio do teste de Shapiro-Wilk, com o intuito de auxiliar a escolha do teste estatístico adequado para efetuar as análises. Realizou-se a estatística descritiva com a finalidade de possibilitar a observação detalhada dos dados, contemplando número de dados, valor mínimo, valor máximo, $1^{\circ}$ e $3^{\circ}$ quartis (percentis 25 e 75\%), média aritmética, mediana, desvio padrão e coeficiente de variação, para cada grupo de faixa populacional.

Além disso, calcularam-se os outliers pelo método exploratório da amplitude interquartis (AIQ) (NAGHETTINI; PINTO, 2007). Todas as ocorrências de outliers foram analisadas individualmente para a decisão sobre sua manutenção ou retirada das análises.

Com relação às análises estatísticas desenvolvidas neste artigo para avaliar os gastos públicos municipais despendidos com os serviços de limpeza urbana e manejo de RS, referentes aos municípios da RMBH no período de 2009 a 2017, o Quadro 2 apresenta os objetivos das análises e o respectivo teste estatístico utilizado.

Destaca-se que se optou pelo teste de Kruskal-Wallis por este testar múltiplas amostras independentes, de modo que verifique se as medianas dos grupos se diferem significativamente, sendo complementado pelo teste de Dunn que indicou entre quais grupos ocorreram as diferenças. Já o teste de correlação de Spearman foi utilizado para avaliar a existência de correlação entre duas variáveis quantitativas. Ambos os testes são não paramétricos dada a ausência de normalidade dos dados, sendo reportada adiante neste texto.

Ressalta-se, também, que o nível de significância adotado para todas as análises estatísticas foi de $5 \%(\alpha=0,05)$ e os pacotes estatísticos utilizados foram os softwares $\mathrm{R}$ (versão 3.4.2) e Statistica (versão 10). Para a melhor interpretação

Quadro 2 - Análises estatísticas realizadas para avaliação dos gastos públicos municipais despendidos com serviços de limpeza urbana, referentes aos municípios da Região Metropolitana de Belo Horizonte , no período de 2009 a 2017.

\begin{tabular}{|c|c|}
\hline Objetivo da análise estatística & $\begin{array}{c}\text { Teste estatístico } \\
\text { utilizado }\end{array}$ \\
\hline $\begin{array}{l}\text { Verificar se há diferenças significativas entre as } \\
\text { despesas com manejo de RSU em função das faixas } \\
\text { populacionais. }\end{array}$ & $\begin{array}{l}\text { Teste de Kruskal-Wallis } \\
\text { Teste de Dunn }\end{array}$ \\
\hline $\begin{array}{l}\text { Avaliar se existe correlação entre a arrecadação } \\
\text { específica e os gastos com os serviços de manejo } \\
\text { de RSU. }\end{array}$ & Correlação de Spearman \\
\hline $\begin{array}{l}\text { Avaliar se existem diferenças significativas entre a } \\
\text { arrecadação específica e os gastos com os serviços } \\
\text { de manejo de RSU, por faixas de população. }\end{array}$ & $\begin{array}{l}\text { Teste de Kruskal-Wallis } \\
\text { Teste de Dunn }\end{array}$ \\
\hline $\begin{array}{l}\text { Avaliar se existe correlação entre as despesas com } \\
\text { os serviços de manejo de RSU e as receitas totais } \\
\text { dos municípios. }\end{array}$ & Correlação de Spearman \\
\hline $\begin{array}{l}\text { Avaliar se existem diferenças significativas entre as } \\
\text { despesas com serviços de manejo de RSU e receitas } \\
\text { totais dos municípios, por faixas de população. }\end{array}$ & $\begin{array}{l}\text { Teste de Kruskal-Wallis } \\
\text { Teste de Dunn }\end{array}$ \\
\hline $\begin{array}{l}\text { Avaliar se existem diferenças significativas entre as } \\
\text { despesas por cada tipo de serviços de manejo de } \\
\text { resíduos sólidos (coleta de RDO' e RPU², coleta de } \\
\text { RSS }^{3} \text {, varrição e outros) e a despesa total com os } \\
\text { serviços de manejo de RSU, por faixas de população. }\end{array}$ & $\begin{array}{l}\text { Teste de Kruskal-Wallis } \\
\text { Teste de Dunn }\end{array}$ \\
\hline
\end{tabular}

${ }^{1}$ Resíduos domésticos; ${ }^{2}$ Resíduos públicos; ${ }^{3}$ Resíduos de serviços de saúde. 
dos dados, elaboraram-se gráficos de barras, de dispersão e Box-Whisker. Portanto as etapas da pesquisa se deram conforme a representação na Figura 2.

\section{RESULTADOS E DISCUSSÃO}

A base para as análises estatísticas foi o Quadro 2, que mostra o teste utilizado em função de cada objetivo.

\section{Teste de normalidade}

Os resultados do teste de normalidade de Shapiro-Wilk apontaram que os dados de gastos e arrecadação relativos ao manejo de RSU, entre 2009 e 2017, não tendem a uma distribuição normal $(\mathrm{p}<0,05)$, ao nível de significância de $5 \%(\alpha=0,05)$.

\section{Avaliação das despesas per capita com manejo de resíduos sólidos urbanos, por faixa de população}

Na Figura 3, são apresentados os gráficos Box-Whisker da variável despesa per capita (R\$) com RSU dos municípios da RMBH, por faixa de habitantes, no período de 2009 a 2017.

A mediana das despesas per capita com RSU na RMBH foi de R\$72,49, sendo o menor valor registrado no grupo "de 5.001 a 10.000 habitantes" (R\$ 48,57) e o maior no grupo "acima de 500.000 habitantes" (R\$ 134,86). O valor médio (R\$78,00) das despesas com RSU na RMBH foi abaixo da referência nacional média para o ano de 2017 (R\$124,44), conforme ABRELPE (2017), e também da referência média mundial para o ano de 2010 ( $\mathrm{R} \$ 122,00)$, de acordo com Hoornweg e Bhada-Tata (2012).

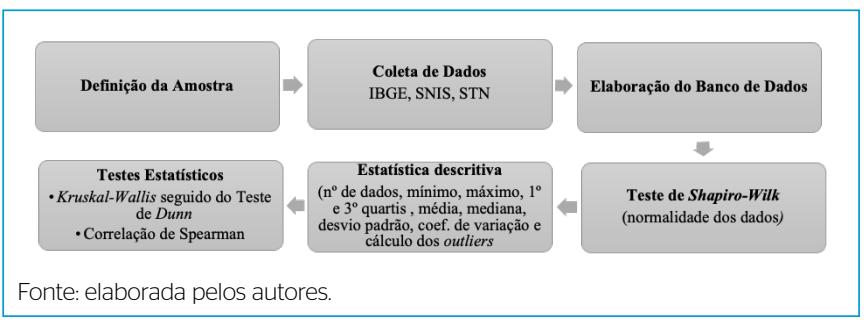

Figura 2 - Fluxograma das etapas da pesquisa.

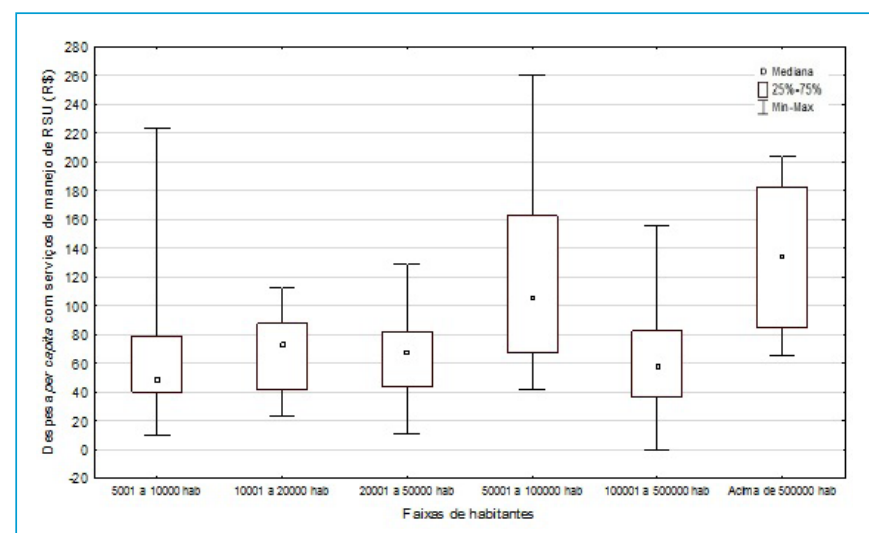

Fonte: elaborada pelos autores, baseado nos dados do Sistema Nacional de Informações Sobre Saneamento (2009 a 2017)

Figura 3 - Despesa per capita ( $\mathrm{R} \$$ ) com resíduos sólidos urbanos dos municípios da Região Metropolitana de Belo Horizonte considerados, por faixas de habitantes, entre 2009 e 2017
Os estudos de Rodrigues, Magalhães Filho e Pereira (2015) e Vasconcelos et al. (2016) avaliaram as variáveis determinantes dos custos da gestão de RSU, sendo elas a influência da população, a presença de coleta seletiva, a existência de empresas públicas ou privadas que realizam os serviços, as condições de relevo, a eficiência produtiva da logística e a produtividade. Os resultados deste estudo apontam como principais determinantes dos custos com RSU a presença da coleta seletiva (realizada por agente privado), a forma de gestão (terceirização integral dos serviços) e a maior densidade populacional. É importante ressaltar que em nenhum desses estudos foi avaliada a eficiência e a cobertura dos serviços ofertados. Segundo dados do SNIS (2017), dos 28 municípios da RMBH que responderam à pesquisa naquele ano, 53,57\% deles declararam possuir programas de coleta seletiva implementados pelas prefeituras.

A Tabela 1 apresenta os resultados dos testes de Kruskal-Wallis e de Dunn, analisando se há diferenças significativas entre as medianas das despesas per capita com RSU, para as diferentes faixas de população testadas. O teste de KruskalWallis, no nível de significância de 5\%, significa que, quando o resultado do valor "p" é menor que 0,05 , há uma diferença significativa entre as medianas dos dados das faixas de habitantes comparadas. Já o teste de Dunn (de comparações múltiplas) identifica em qual par de faixas de habitantes comparado está a diferença estatisticamente significativa (valores destacados em negrito na Tabela 1).

Com base nos resultados apresentados na Tabela 1 e na Figura 3, é possível notar a existência de diferença significativa das despesas per capita com RSU nas faixas de habitantes da RMBH, ao nível de significância de 5\%. Destaca-se o grupo de municípios "acima de 500.000 habitantes" cuja mediana difere-se significativamente de todos os outros grupos, exceto do grupo "de 50.001 a 100.000 habitantes".

Vasconcelos et al. (2016) estudaram os custos com RSU em 227 municípios do estado do Paraná e concluíram que o aumento na densidade populacional é um dos fatores determinantes dos custos, em que o aumento da população pode indicar aumento da cadeia logística para realizar a coleta, do número de profissionais contratados e da área total do município para realizar a varrição.

Esse resultado contradiz a percepção de que municípios de maior porte geram ganhos de economia de escala, como proposto por Massukado et al (2013). Segundo Jacobi e Besen (2011), alguns dos motivos da perda de economia de escala em municípios de grande porte podem estar relacionados com a localização das áreas reservadas ao destino final dos RSU, que geralmente são distantes dos locais habitados - onde os resíduos são coletados. Ainda sobre a economia de escala na prestação de serviços de manejo de RSU, Simões e Marques (2012) avaliaram estudos de diferentes autores, realizados nos Estados Unidos e também na Europa, e concluíram que não há consenso sobre a escala ótima de população para os serviços de RSU.

\section{Correlação entre arrecadação específica com os serviços de resíduos sólidos urbanos e gastos relativos a esses serviços}

Visando analisar a sustentabilidade financeira das despesas municipais com RSU e a receita específica arrecadada por meio de taxas e tarifas para gestão e manejo de RSU, ambas per capita, para a RMBH, no período de 2009 a 2017, efetuou-se o teste de correlação de Spearman com essas duas variáveis, e o resultado sugere a existência de correlação $(p<0,05)$. $O$ valor do coeficiente 
Tabela 1 - Comparação estatística das despesas per capita com manejo de resíduos sólidos urbanos por faixas de habitantes, referentes aos municípios da Região Metropolitana de Belo Horizonte, no período de 2009 a 2017*.

\begin{tabular}{|c|c|c|c|c|c|c|}
\hline \multicolumn{7}{|c|}{ Existe diferença significativa entre as seis faixas de habitantes avaliadas } \\
\hline \multicolumn{7}{|c|}{$(p=0,000)$} \\
\hline \multicolumn{7}{|c|}{ Comparações Múltiplas } \\
\hline Faixas de hab. & 5.001 a 10.000 & 10.001 a 20.000 & 20.001 a 50.000 & 50.001 a 100.000 & 100.001 a 500.000 & Acima 500.000 \\
\hline 5.001 a 10.000 & & 1,000000 & 1,000000 & 0,000574 & 1,000000 & 0,000014 \\
\hline 10.001 a 20.000 & 1,000000 & & 1,000000 & 0,123195 & 1,000000 & 0,007304 \\
\hline 20.001 a 50.000 & 1,000000 & 1,000000 & & 0,003782 & 1,000000 & 0,000095 \\
\hline 50.001 a 100.000 & 0,000574 & 0,123195 & 0,003782 & & 0,000490 & 1,000000 \\
\hline 100.001 a 500.000 & 1,000000 & 1,000000 & 1,000000 & 0,000490 & & 0,000010 \\
\hline Acima 500.000 & 0,000014 & 0,007304 & 0,000095 & 1,000000 & 0,000010 & \\
\hline
\end{tabular}

*Entre parênteses: probabilidade calculada pelo software; valores destacados em negrito: faixas populacionais que apresentaram diferença significativa.

da correlação encontrado foi de $r=0,364$ que, de acordo com Callegari-Jacques (2003), é uma correlação regular. Não foi avaliada a correlação entre as variáveis em cada faixa de habitantes, uma vez que o porte "acima de 500.000" é composto de apenas dois municípios e, portanto, apresenta um número muito reduzido de dados para inferir a existência de correlação. Na Figura 4, tem-se o gráfico de dispersão dessas duas variáveis, em que somente $2,75 \%$ dos dados informados mostram autossuficiência financeira, ou seja, contam com despesas compatíveis com a arrecadação. Destaca-se um ponto em que a arrecadação per capita $(\mathrm{R} \$ 264,15)$ foi muito maior do que a despesa ( $\mathrm{R} \$ 155,25)$.

A PNRS (BRASIL, 2010) e também a PERS (MINAS GERAIS, 2009) têm como princípio a sustentabilidade operacional e financeira para regularidade, continuidade, funcionalidade e universalização da prestação de serviços públicos de limpeza urbana e manejo de resíduos sólidos, com a adoção de mecanismos gerenciais e econômicos que assegurem a recuperação dos custos dos serviços prestados. Os dados do SNIS (2017) apontam uma situação controversa, em que 46,3\% dos municípios brasileiros que responderam à pesquisa não praticavam, à época, a cobrança pelos serviços de coleta domiciliar, transporte e destinação final. Entre os 31 municípios estudados na RMBH, apenas 7 (22,58\%) ainda não faziam a cobrança pelos referidos serviços (SNIS, 2017).

\section{Avaliação da proporção entre a arrecadação específica e os gastos com os serviços de manejo de resíduos sólidos urbanos, por faixas de população}

Na Figura 5, apresenta-se o gráfico Box-Whisker do percentual das despesas per capita com manejo de RSU em relação às receitas per capita arrecadadas anualmente com taxas e tarifas referentes à gestão de RSU, no período de 2009 a 2017, de acordo com as faixas de população.

Para avaliar a existência de diferenças significativas entre as medianas dos referidos percentuais, utilizou-se o teste de Kruskal-Wallis, e o resultado $(\mathrm{p}=0,1625)$ demonstra que não há diferença significativa entre as medianas dos dados analisados.

Para analisar o gráfico Box-Whisker apresentado na Figura 5 e o resultado do teste de Kruskal-Wallis, optou-se por remover os outliers das variáveis em questão. Os resultados permitem identificar que não há diferenças significativas no percentual das despesas com manejo de RSU em relação

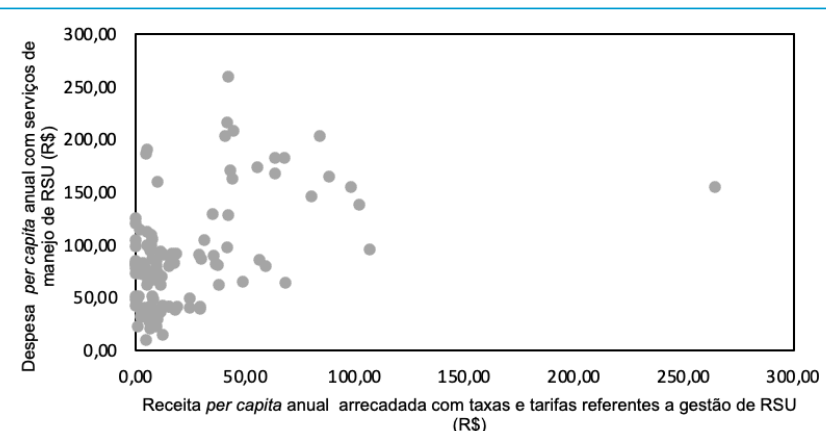

Fonte: elaborado pelos autores, baseado nos dados do Sistema Nacional de Informações Sobre Saneamento (2009 a 2017).

Figura 4 - Despesa per capita anual (R\$) com serviços de manejo de resíduos sólidos urbanos versus receita per capita anual ( $R$ \$) arrecadada com taxas e tarifas referentes à gestão de resíduos sólidos urbanos, 2009 a 2017, na Região Metropolitana de Belo Horizonte.

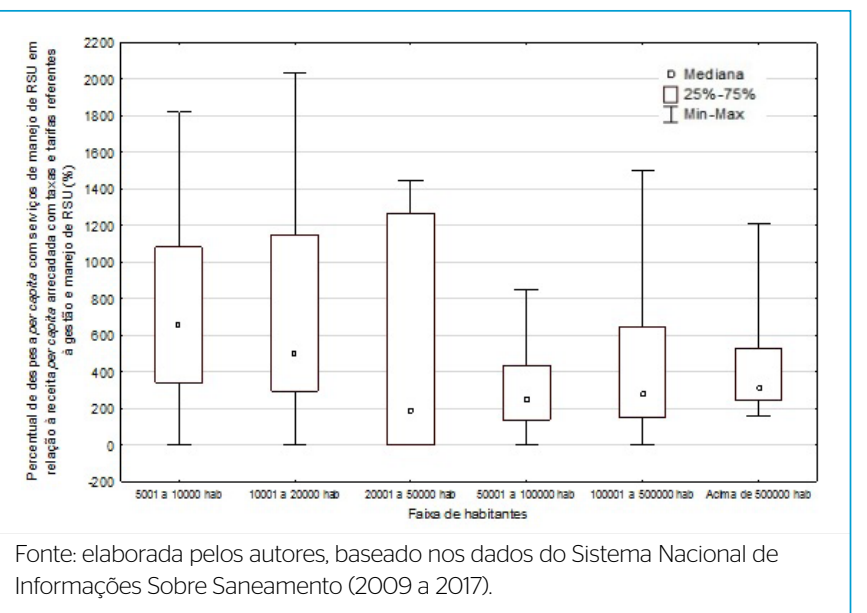

Figura 5 - Percentual das despesas per capita com manejo de resíduos sólidos urbanos em relação às receitas per capita arrecadadas anualmente com taxas e tarifas referentes à gestão de resíduos sólidos urbanos, em função das faixas de população dos municípios da Região Metropolitana de Belo Horizonte, no período de 2009 a 2017.

às receitas per capita arrecadadas anualmente com taxas e tarifas referentes à gestão de RSU, quando analisados os municípios da RMBH por faixas de habitantes. 


\section{Avaliação da existência de correlação entre as despesas com resíduos sólidos urbanos e as receitas totais dos municípios}

Para avaliar a existência de correlação entre as despesas com os serviços de manejo de RSU e as receitas totais dos municípios da RMBH, realizou-se o teste de Spearman. Com base no resultado do teste, é possível inferir a existência de correlação entre as duas variáveis $(\mathrm{p}<0,05)$, no nível de significância de 5\%. A análise qualitativa da intensidade da correlação, de acordo com metodologia proposta por Callegari-Jacques (2003), sugere uma correlação regular $(r=0,552)$. Novamente, ressalta-se que não foi avaliada a correlação entre as variáveis dentro de cada faixa de habitantes por falta de dados suficientes.

A Figura 6 apresenta o gráfico de dispersão entre essas variáveis. É possível perceber uma concentração dos dados, refletindo que grande parte dos municípios $(93,64 \%)$ tem despesas anuais per capita com manejo de RSU abaixo de R $\$ 100$, destes, $96,91 \%$ possuem receita total anual per capita de até $\mathrm{R} \$ 3.000$.

\section{Avaliação da proporção entre as despesas com serviços de manejo de resíduos sólidos urbanos e receitas totais dos municípios, por faixas de população}

$\mathrm{Na}$ Figura 7, observam-se os gráficos Box-Whisker do percentual entre a despesa per capita com manejo de RSU em relação à receita total per capita dos municípios da RMBH, de acordo com as faixas de população. A figura mostra que as medianas das despesas referentes ao manejo de RSU nos municípios da $\mathrm{RMBH}$, para todas as faixas populacionais, estão compreendidas entre a faixa de 2 e $4 \%$ do total dos recursos municipais.

Segundo dados do Instituto de Pesquisas Tecnológicas (IPT, 2000) e do Compromisso Empresarial para Reciclagem (CEMPRE, 2018), no Brasil, de forma geral, os gastos com serviços de limpeza pública significam entre 5 e 15\% dos recursos do orçamento municipal. Dessa forma, as medianas dos municípios da RMBH encontram-se abaixo da média nacional; no entanto, é possível perceber a existência de valores na faixa citada na literatura.

Para avaliar se existem diferenças significativas entre os percentuais das despesas per capita com manejo de RSU em relação às receitas totais per capita dos municípios da RMBH, em função das faixas de habitantes, utilizaram-se o

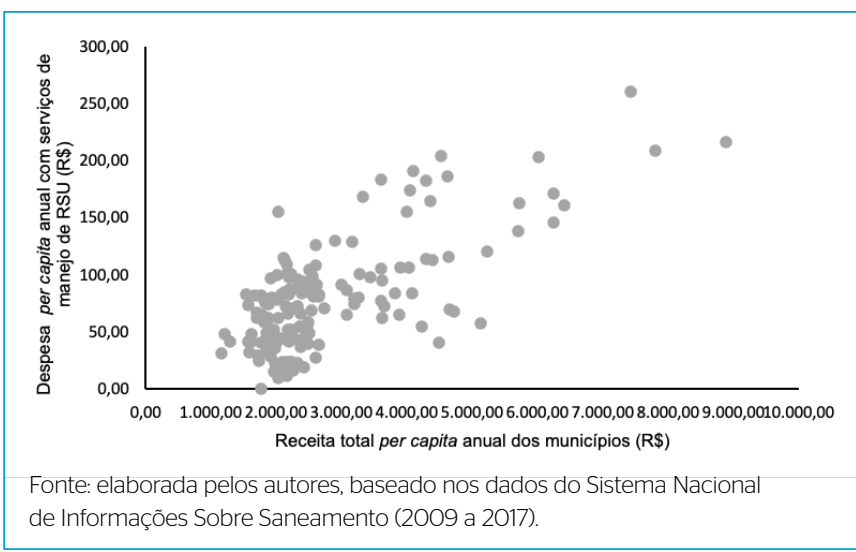

Figura 6 - Despesa per capita anual com serviços de manejo de resíduos sólidos urbanos versus receita total anual per capita dos municípios da Região Metropolitana de Belo Horizonte, no período entre 2009 a 2017. teste de Kruskal-Wallis e o teste de comparações múltiplas. Os resultados desses testes estão apresentados na Tabela 2, no nível de significância de 5\%, conforme já mencionado na Tabela 1.

Com base nos resultados da Tabela 2 e da Figura 7, é possível notar a existência de diferença significativa no percentual entre as despesas com manejo de RSU em relação às receitas totais dos municípios, no nível de significância de 5\%. A mediana do grupo de municípios "acima de 500.000 habitantes" foi a única que diferiu significativamente das medianas de outros grupos: "de 5.001 a 10.000 hab.", "de 20.001 a 50.000 hab." e "de 100.001 a 500.000 hab." A faixa "acima de 500.000 hab." foi a com maior porte populacional analisada no estudo, e Silva et al. (2015) ressaltam que os municípios e os estados brasileiros que têm uma estrutura urbana muito grande possuem maior complexidade da gestão da cadeia de resíduos sólidos, o que representa uma parcela expressiva dos gastos municipais.

\section{Avaliação das proporções entre as despesas por cada tipo de serviço de limpeza pública e do manejo de resíduos sólidos e a despesa total com os serviços de limpeza pública e o manejo de resíduos sólidos urbanos, por faixas de população}

A Figura 8 apresenta o percentual de contribuição das despesas por cada tipo de serviço de limpeza pública e do manejo de RS (coleta de resíduos domiciliares [RDO] e de resíduos sólidos públicos [RPU], coleta de resíduos de serviços de saúde [RSS], varrição e outros) em relação aos gastos totais municipais com RSU, no período de 2009 a 2017. Os municípios foram agrupados por faixas de habitantes.

O percentual das despesas referente aos "outros" serviços ofertados pelo município é expressivo em relação aos demais serviços especificados. Não foi possível segregar este grupo para avaliar o custo pormenorizado, pois se tratam de dados secundários não disponibilizados, mas vale destacar que o custo com a disposição final dos RSU está inserido nesse grupo, o que pode explicar o alto valor percentual desse item na composição dos custos.

Ainda sobre o valor percentual das despesas com o serviço de coleta de RDO e RPU, o menor valor percentual $(27,8 \%)$ foi observado para a faixa

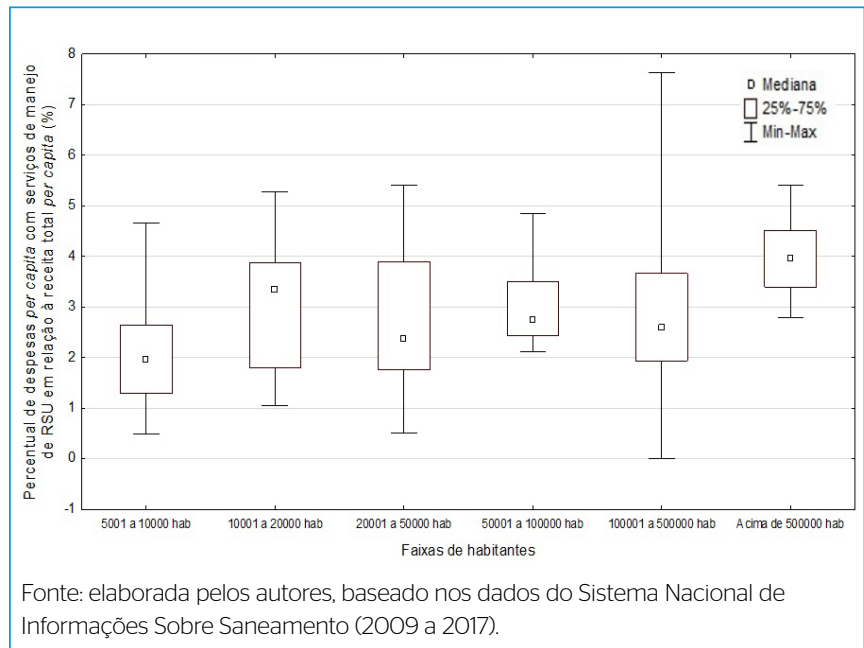

Figura 7 - Percentual entre a despesa per capita com manejo de resíduos sólidos urbanos em relação à receita total per capita dos municípios anuais, em função das faixas de habitantes dos municípios da Região Metropolitana de Belo Horizonte, no período entre 2009 a 2017. 
Tabela 2 - Comparação estatística percentual das despesas per capita com manejo de resíduos sólidos urbanos sobre as receitas totais per capita dos municípios da Região Metropolitana de Belo Horizonte, no período de 2009 a 2017, com os grupos de portes populacionais*.

\begin{tabular}{|c|c|c|c|c|c|c|}
\hline \multicolumn{7}{|c|}{ Existe diferença significativa entre as seis faixas de habitantes avaliadas $(p=0,000)$} \\
\hline \multicolumn{7}{|c|}{ Comparações Múltiplas } \\
\hline Faixas de hab. & 5.001 a 10.000 & 10.001 a 20.000 & 20.001 a 50.000 & 50.001 a 100.000 & 100.001 a 500.000 & Acima 500.000 \\
\hline 5.001 a 10.000 & & 0,109772 & 1,000000 & 0,180788 & 0,861498 & 0,000005 \\
\hline 10.001 a 20.000 & 0,109772 & & 1,000000 & 1,000000 & 1,000000 & 0,178545 \\
\hline 20.001 a 50.000 & 1,000000 & 1,000000 & & 1,000000 & 1,000000 & 0,001273 \\
\hline 50.001 a 100.000 & 0,180788 & 1,000000 & 1,000000 & & 1,000000 & 0,187995 \\
\hline 100.001 a 500.000 & 0,861498 & 1,000000 & 1,000000 & 1,000000 & & 0,002936 \\
\hline Acima 500.000 & 0,000005 & 0,178545 & 0,001273 & 0,187995 & 0,002936 & \\
\hline
\end{tabular}

*Entre parênteses: probabilidade calculada pelo software; valores destacados em negrito: faixas populacionais que apresentaram diferença significativa.

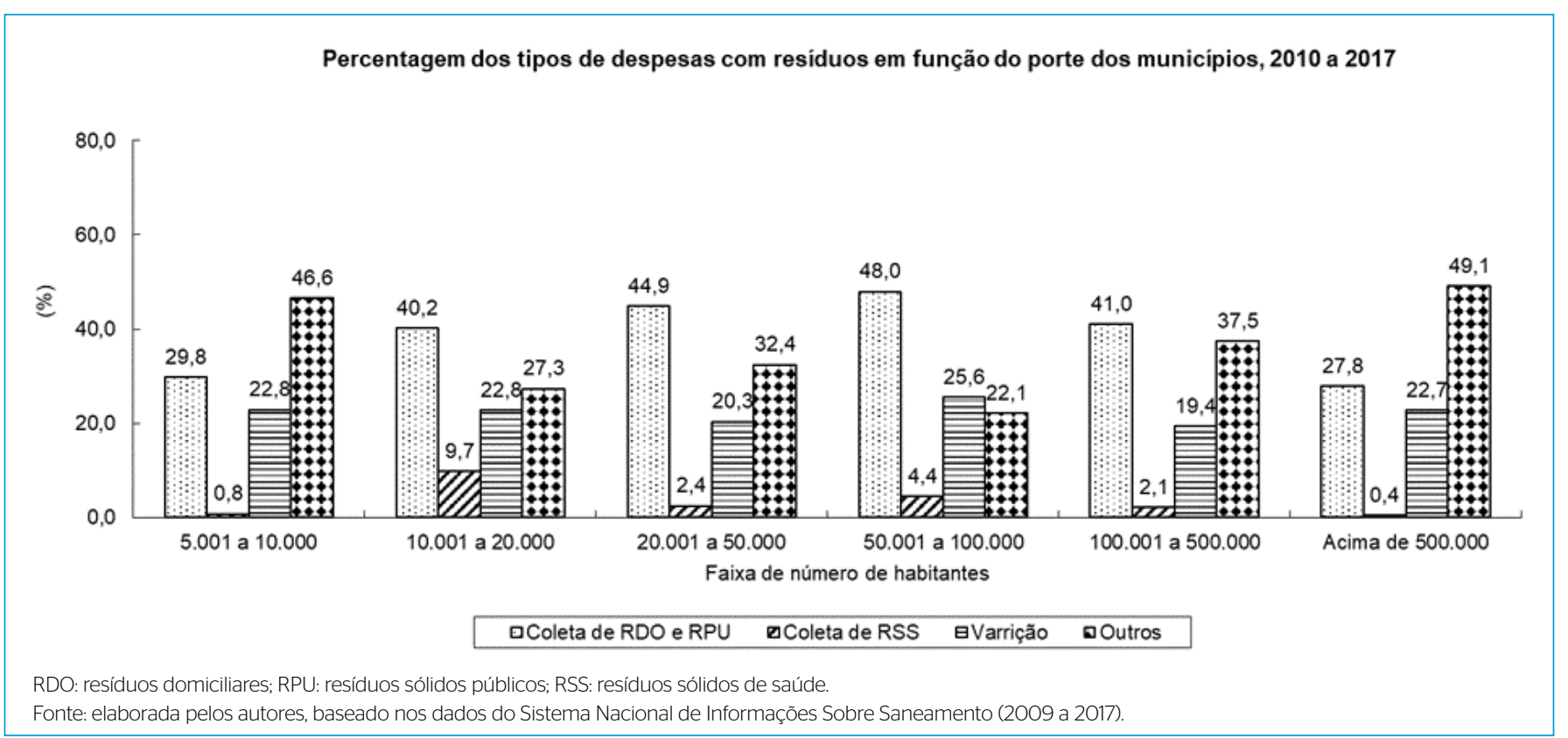

Figura 8 - Percentual dos tipos de despesas com resíduos sólidos dos municípios da Região Metropolitana de Belo Horizonte, por faixas de habitantes, entre 2009 a 2017.

“acima de 500.000 hab.". A literatura evoca o fator de escala, em que, de uma maneira geral, quanto maior o volume da prestação do serviço menor o custo, mas, novamente, cabe salientar que é necessário cautela nessa avaliação, pois, de acordo com Simões e Marques (2012), não há consenso sobre a escala ótima de serviços de RS.

$\mathrm{Na}$ Figura 9, apresentam-se os gráficos Box-Whisker referentes à proporção entre as despesas com cada tipo de serviço de manejo de RS prestado e a despesa total com os serviços de manejo de RSU dos municípios da RMBH, agrupados por faixas de habitantes.

Os resultados dos testes de Kruskal-Wallis e de comparações múltiplas para avaliar a proporção entre cada tipo de serviço de manejo de RS e a despesa total com o manejo de RSU, por faixas de habitantes, estão na Tabela 3.

Com base nos resultados da Tabela 3 e da Figura 9, é possível notar que a faixa "acima de 500.000 hab." difere-se significativamente dos demais grupos para a maioria dos serviços de manejo de resíduos avaliados, no nível de significância de 5\%. Para o serviço de coleta de RDO e RPU, o percentual da despesa dessa faixa de municípios foi significativamente inferior ao de todas as outras faixas de habitantes. Já para o serviço de coleta de RSS, a faixa "acima de 500.000 hab." só não divergiu significativamente da faixa populacional "de 10.001 a 20.000 hab., sendo inferior a todas as outras faixas de habitantes, no nível de significância de $5 \%$.

Para os "outros serviços" de manejo de RS, novamente os resultados dos municípios com faixa de população acima de 500 mil habitantes foram significativamente superiores aos de todas as outras faixas de habitantes, no nível de significância de 5\%. Somente para o serviço de "varrição" de RS o grupo não apresentou diferença significativa, no nível de $5 \%$, sendo a diferença significativa para esse serviço observada entre as faixas de habitantes "de 5.001 a 10.000" e "de 100.001 a 500.000", para qual esta última foi significativamente inferior, no nível de significância de $5 \%$.

Os valores destacados em negrito, na Tabela 3, evidenciam os pontos em que os resultados dos testes apresentam diferença estatisticamente significativa entre as medianas avaliadas ao nível de $5 \%$, ou seja, em que o "p" valor é menor que 0,05 . Valem as explicações para o valor de "p" dadas nas Tabelas 1 e 2 . 

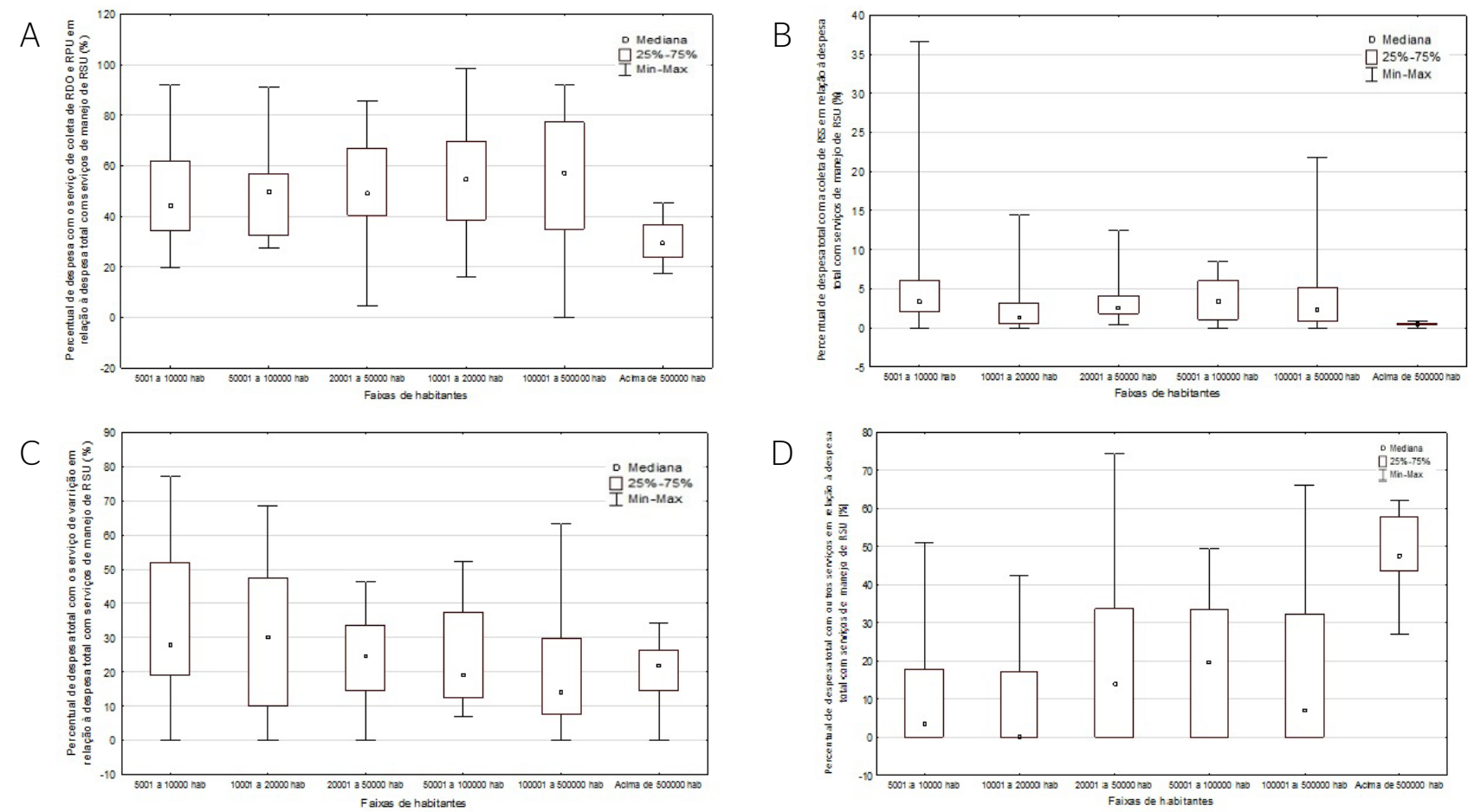

RDO: resíduos domiciliares; RPU: resíduos sólidos públicos; RSS: resíduos sólidos de saúde.

Fonte: elaborada pelos autores, baseado nos dados do Sistema Nacional de Informações Sobre Saneamento (2009 a 2017).

Figura 9 - Proporção entre as despesas com cada tipo de serviço de manejo de resíduos sólidos prestado e a despesa total com os serviços de manejo de resíduos sólidos urbanos dos municípios da Região Metropolitana de Belo Horizonte, agrupados por faixas de habitantes, no período de 2009 a 2017 . (A) Serviço de coleta de resíduos domiciliares e de resíduos sólidos públicos; (B) serviço de coleta de resíduos de serviços de saúde; (C) serviço de varrição de resíduos sólidos; (D) outros serviços de manejo de resíduos sólidos urbanos.

Tabela 3 - Comparação estatística da proporção entre a despesa com cada tipo de serviço de manejo de resíduos sólidos e a despesa total com serviços de manejo de resíduos sólidos urbanos dos municípios da Região Metropolitana de Belo Horizonte, no período de 2009 a 2017, de acordo com as faixas de habitantes*.

\begin{tabular}{|c|c|c|c|c|c|c|}
\hline \multirow{4}{*}{\multicolumn{3}{|c|}{ Faixas de habitantes }} & \multicolumn{4}{|c|}{$\begin{array}{l}\text { Existe diferença significativa entre as seis faixas de } \\
\text { habitantes avaliadas para cada tipo de serviços de manejo de RSU }\end{array}$} \\
\hline & & & $\begin{array}{l}\text { Coleta de resíduos } \\
\text { sólidos domiciliares e } \\
\text { públicos }\end{array}$ & $\begin{array}{l}\text { Coleta de resíduos } \\
\text { sólidos de saúde }\end{array}$ & $\begin{array}{l}\text { Varrição de } \\
\text { resíduos sólidos }\end{array}$ & $\begin{array}{l}\text { Outros serviços serviço } \\
\text { de manejo de RSU }\end{array}$ \\
\hline & & & $(p=0,0006)$ & $(p=0,000)$ & $(p=0,0134)$ & $(p=0,0000)$ \\
\hline & & & \multicolumn{4}{|c|}{ Comparações múltiplas } \\
\hline 5.001 a 10.000 & vS. & 20.001 a 50.000 & $(p=1,000000)$ & $(p=1,000000)$ & $(p=1,000000)$ & $(p=1,000000)$ \\
\hline 5.001 a 10.000 & vS. & 50.001 a 100.000 & $(p=1,000000)$ & $(p=1,000000)$ & $(p=1,000000)$ & $(p=1,000000)$ \\
\hline 5.001 a 10.000 & vS. & 10.001 a 20.000 & $(p=1,000000)$ & $(p=0,361402)$ & $(p=1,000000)$ & $(p=1,000000)$ \\
\hline Acima 500.000 & vs. & 100.001 a 500.000 & $(p=0,000226)$ & $(p=0,000131)$ & $(p=1,000000)$ & $(p=0,000112)$ \\
\hline Acima 500.000 & vS. & 20.001 a 50.000 & $(p=0,000947)$ & $(p=0,000021)$ & $(p=1,000000)$ & $(p=0,000316)$ \\
\hline 100.001 a 500.000 & vs. & 50.001 a 100.000 & $(p=1,000000)$ & $(p=1,000000)$ & $(p=1,000000)$ & $(p=1,000000)$ \\
\hline 100.001 a 500.000 & vs. & 10.001 a 20.000 & $(p=1,000000)$ & $(p=1,000000)$ & $(p=0,385161)$ & $(p=1,000000)$ \\
\hline 20.001 a 50.000 & vs. & 50.001 a 100.000 & $(p=1,000000)$ & $(p=1,000000)$ & $(p=1,000000)$ & $(p=1,000000)$ \\
\hline 20.001 a 50.000 & vs. & 10.001 a 20.000 & $(p=1,000000)$ & $(p=1,000000)$ & $(p=1,000000)$ & $(p=0,787428)$ \\
\hline 50.001 a 100.000 & vs. & 10.001 a 20.000 & $(p=1,000000)$ & $(p=1,000000)$ & $(p=1,000000)$ & $(p=1,000000)$ \\
\hline
\end{tabular}

*Entre parênteses: probabilidade calculada pelo software; valores destacados em negrito: faixas populacionais que apresentaram diferença significativa; RSU: resíduos sólidos urbanos 


\section{CONCLUSÕES}

Os resultados deste estudo de caso apontaram diferenças significativas do custo per capita despendido pelos municípios com todos os serviços de manejo de resíduos sólidos, entre as seis faixas populacionais comparadas. O custo correspondente à faixa "acima de 500.000 hab.", no geral, foi significativamente superior aos valores das outras faixas de habitantes.

Quando analisado cada tipo de serviço de manejo de resíduo prestado pelos municípios (coleta de RDO e de RPU, coleta de RSS, varrição e outros tipos de serviços), foi possível concluir que a faixa "acima de 500.000 hab." apresenta valores diferentes significativamente das demais faixas de habitantes. No caso dos serviços de coleta de RDO e RPU e da coleta de RSS, a referida faixa foi significativamente inferior; já para os "outros" serviços foi significativamente superior às demais faixas de habitantes.

Somente no serviço de varrição é que, em geral, as despesas com o serviço não demonstraram diferença significativa entre as faixas de habitantes. As únicas faixas significativamente diferentes foram as "de 5.001 a 10.000 hab." e "de 100.001 a 500.000 hab."

A análise do percentual das despesas com manejo de RSU sobre as receitas totais dos municípios mostrou, novamente, uma diferença significativa entre a faixa "acima de 500.000 hab." e as demais faixas, que não possuem diferenças significativas entre si em relação a essa variável.

Sobre a sustentabilidade financeira, também chamada de autossuficiência, analisou-se o percentual entre as despesas per capita com manejo de RSU em relação às receitas per capita arrecadadas anualmente com taxas e tarifas referentes à gestão de RSU, sendo permitido inferir que, no nível de significância de 5\%, em todas as faixas de habitantes não houve diferença significativa.
A existência de poucos estudos relacionando as temáticas "custos e resíduos", tanto nacional quanto internacionalmente, apresenta-se como um dificultador para comparar e entender o comportamento dos gastos públicos. As informações de municípios e países com realidade de gestão e tecnologias muito diferentes dos municípios estudados, sobretudo os dados internacionais, devem ser usados com cautela para comparação.

A principal fonte de dados utilizados para os valores financeiros, SNIS, trata-se de dados autodeclarados pelos municípios, e a sua análise detalhada permitiu identificar pontos de atenção que possam ter sido erros de entendimento. Sugere-se, portanto, uma análise mais aprofundada, incluindo pesquisa de campo para alinhamento e checagem desses dados. Além disso, o banco de dados elaborado com base nas informações do SNIS apresentou um número reduzido de dados dependendo da análise realizada.

E, por fim, este estudo teve como objetivo geral identificar um panorama da situação dos gastos com os serviços de manejo de RSU para os municípios da RMBH segregados por faixas de habitantes, contribuindo para ampliar a discussão sobre o tema e fomentando os escassos estudos sobre custos da gestão de RSU. É relevante destacar que o estudo é limitado no que se refere a avaliações sobre a qualidade e a cobertura dos serviços prestados e o confronto com os respectivos custos. Para futuros trabalhos, sugere-se avaliar os fatores determinantes dos custos per capita com os serviços de manejo de resíduos, permitindo, assim, uma análise completa do cenário.

\section{CONTRIBUIÇÕES DOS AUTORES}

Ferreira, A. C. N.: Escrita — Primeira Redação, Investigação, Metodologia, Administração do Projeto. Barros, R. T. V.: Supervisão, Validação, Escrita - Revisão e Edição.

\section{REFERÊNCIAS}

AGÊNCIA DA REGIÃO METROPOLITANA DE BELO HORIZONTE (RMBH). Portal Eletrônico. RMBH, 2018. Disponível em: http://www.agenciarmbh. mg.gov.br/mapa-conheca-os-municipios/. Acesso em: 2 jun. 2018.

ASSOCIAÇÃO BRASILEIRA DE EMPRESAS DE LIMPEZA PÚBLICA E RESÍDUOS ESPECIAIS (ABRELPE). Panorama dos resíduos sólidos no Brasil 2017. 15. ed. São Paulo: Abrelpe, 2017. 74 p.

BARROS, R.T.V. Elementos de resíduos sólidos. Belo Horizonte: Tessitura, 2012. $424 \mathrm{p}$

BARROS, R.T.V.; SILVA, T.A.S.; MIRANDA, T.G. O gerenciamento dos resíduos verdes na UFMG: outras possibilidades. Belo Horizonte: UFMG, 2005.

BELO HORIZONTE. Plano Municipal de Saneamento de Belo Horizonte: 2016/2019. Belo Horizonte: Prefeitura de Belo Horizonte, 2016.

BRASIL. Lei no 12.305, de 2 de agosto de 2010. Institui a política nacional de resíduos sólidos; altera a Lei no 9.605, de 12 de fevereiro de 1998; e dá outras providências. Diário Oficial da União, Brasília, Seção 1, p. 3, 3 ago. 2010.

CALLEGARI-JACQUES, S. Bioestatística: princípios e aplicações. Porto Alegre: Artmed, 2003. 255 p.
COMPROMISSO EMPRESARIAL PARA RECICLAGEM (CEMPRE). Lixo Municipal: Manual do Gerenciamento Integrado. 3. ed. São Paulo: CEMPRE, 2018. 310 p.

CONCEIÇÃO, M.G.; FERREIRA, A.S.; GOMES, S.M.; RIBEIRO, L.W.S.; JESUS RIBEIRO, R. A produção científica em contabilidade sobre resíduos sólidos no Brasil: Um estudo bibliométrica. In: ENGEMA, 14., 2012, São Paulo. Anais.. São Paulo, 2012.

CONCEIÇÃO, M.G.; FERREIRA, A.S.; GOMES, S.M.; RIBEIRO, L.W.S.; JESUS RIBEIRO, R. Perfil e evolução da pesquisa em gestão de resíduos sólidos: uma análise bibliométrica. In: CONGRESSO BRASILEIRO DE CONTABILIDADE, 2O., 2016, Fortaleza. Anais... Fortaleza, 2016.

FUNDAÇÃO ESTADUAL DO MEIO AMBIENTE (FEAM). Classificação e Panorama da Destinação dos Resíduos Sólidos Urbanos em Minas Gerais. Minas Gerais: FEAM, 2016. Disponível em: http://www.feam.br/images/ stories/2017/RESIDUOS/Minas_Sem_Lixoes/Classifica\%C3\%A7\%C3\%A3o_e_ Panorama_Final_2016_para_ASCOM.pdf. Acesso em: 9 jun. 2019.

GONÇALVES, M.A.; TANAKA, A.K.; AMEDOMAR, A. A destinação final dos resíduos sólidos urbanos: alternativas para a cidade de São Paulo através de casos de sucesso. Future Studies Research Journal, v. 5, n. 1, p. 96-129, 2013. https://doi.org/10.24023/FutureJournal/2175-5825/2013.v5i1.112 
Hoornweg, D.; Bhada-Tata, P. What a waste: a global review of solid waste management. Washington, D.C.: World Bank, 2012. 116 p.

INSTITUTO BRASILEIRO DE GEOGRAFIA E ESTATISTICA (IBGE). Projeções da População 2007-2019. IBGE. Disponível em: https://www.ibge.gov.br/ estatisticas/sociais/populacao/9109-projecao-da-populacao.html?=\&t=oque-e. Acesso em: 20 maio 2019.

Instituto de Pesquisas Tecnológicas (IPT). Lixo Municipal: Manual do Gerenciamento Integrado. 2. ed. São Paulo: CEMPRE, 2000. 310 p.

JACOBI, P.R.; BESEN, G.R. Gestão de resíduos sólidos em São Paulo: desafios da sustentabilidade. Estudos Avançados, v. 25, n. 71, p. 135-158, 2011. https:// doi.org/10.1590/S0103-40142011000100010

MASSUKADO, L.M.; MILANEZ, B.; LUEDEMANN, G.; HARGRAVE, J. Diagnóstico da Gestão de resíduos sólidos urbanos no Brasil: uma análise pós PNSB 2008 - ênfase na destinação final e nos resíduos orgânicos. Revista DAE, n. 192, p. 22-33, 2013. http://dx.doi.org/10.4322/dae.2014.105

MINAS GERAIS. Lei no 6.303, de 30 de abril de 1974. Dispõe sobre a Região Metropolitana de Belo Horizonte e dá outras providências. Diário do Executivo de Minas Gerais, Belo Horizonte, 30 abr. 1974.

MINAS GERAIS. Lei no 18.031/09, de 12 de janeiro de 2009: Política Estadual de Resíduos Sólidos. Diário Oficial da União, Belo Horizonte, 12 jan. 2009. 26 p. Disponível em: http://www.siam.mg.gov.br/sla/download. pdf?idNorma=9272. Acesso em: 11 jan. 2019.

MINAS GERAIS. Plano metropolitano de resíduos sólidos: região metropolitana de Belo Horizonte e colar metropolitano (PMRS). Belo Horizonte: Secretaria Extraordinária de Gestão Metropolitana, 2013.
Naghettini, M.; Pinto, E.J.A. Hidrologia Estatística. Belo Horizonte: CPRM, 2007. $552 \mathrm{p}$.

RODRIGUES, W.; MAGALHÃES FILHO, L.N.L.; PEREIRA, R. Análise dos Determinantes dos custos de resíduos sólidos urbanos nas capitais estaduais brasileiras. Revista Brasileira de Gestão Urbana, v. 8, n. 1, p. 130-141, 15 dez. 2015. http://dx.doi.org/10.1590/2175-3369.008.001.ao02

SECRETARIA DO TESOURO NACIONAL (STN). Finanças Municipais. STN, 2019. Disponível em: https://www.gov.br/tesouronacional/pt-br. Acesso em: 20 maio 2019.

SILVA, C.L.; FUGGI, G.M.; BASSI, N.S.S.; SANTOYO, A.H. O que é relevante para planejar e gerir resíduos sólidos? Uma proposta de definição de variáveis para a formulação e avaliação de políticas públicas. Revista Bibliográfica de Geografía y Ciencias Sociales, v. 20, n. 1114, p. 1-25, 2015. https://doi. org/10.1344/b3w.0.2015.26103

SIMÕES, P.; MARQUES, R.C. On the economic performance of the waste sector. A literature review. Journal of Environmental Management, v. 106, p. 40-47, 2012. https://doi.org/10.1016/j.jenvman.2012.04.005

SISTEMA NACIONAL DE INFORMAÇÕES SOBRE SANEAMENTO (SNIS). Diagnóstico do manejo de Resíduos Sólidos Urbanos - 2015. SNIS, 20092019. Disponivel em http://www.snis.gov.br/diagnostico-residuos-solidos/ diagnostico-rs-20140. Acesso em: 24 maio 2020.

VASCONCELOS, M.C.; BACH, T.M.; MATIOLLO, D.; JUNIOR, J.; SOUZA, A. SILVA, W.V. Evidências relativas aos Custos de Resíduos Sólidos Urbanos Municipais: uma Análise dos Fatores Determinantes. In: CONGRESSO BRASILEIRO DE CUSTOS, 23., 2016, Porto de Galinhas. Anais... Porto de Galinhas, 2016. p. 16-18. 